\title{
ESTUDO RETROSPECTIVO DE 53 CRIANÇAS COM DOENÇA DE HIRSCHSPRUNG: ACHADOS CLÍNICOS E LABORATORIAIS
}

\author{
RETROSPECTIVE STUDY OF 53 CHILDREN WITH HIRSCHSPRUNG 'S DISEASE: \\ CLINICAL AND LABORATORIAL FINDINGS
}

Rosa H M Bigélli'; Maria I M Fernandes²; Lívia C Galvão² \& Regina Sawamura ${ }^{1}$

\begin{abstract}
${ }^{1}$ Médica Assistente. ${ }^{2}$ Docente. Departamento de Puericultura e Pediatria - Setor de Gastroenterologia Pediátrica. Faculdade de Medicina de Ribeirão Preto - USP.

CoRRESPONDÊNCIA: Maria Inez Machado Fernandes. Departamento de Puericultura e Pediatria da FMRP/USP. Hospital das Clínicas de Ribeirão Preto. Av. Bandeirantes, 3900 - CEP 14049-900 Ribeirão Preto - SP. Fone: (016) 6022573 - Fax: (016) 602-2700 E-mail: mimferna@fmrp.usp.br
\end{abstract}

BIGÉLLI RHM; FERNANDES MIM; GALVÃO LC \& SAWAMURA R. Estudo retrospectivo de 53 crianças com doença de Hirschsprung: achados clínicos e laboratoriais. Medicina, Ribeirão Preto, 35: 78-84, jan./mar. 2002.

RESUMO: Objetivo: analisar algumas características clínicas e laboratoriais de crianças com doença de Hirschsprung. Métodos: estudo retrospectivo de 53 crianças com diagnóstico final de doença de Hirschsprung, atendidas de janeiro de 1981 a dezembro de 1999, no Hospital das Clínicas/ USP, Ribeirão Preto. Foram analisados alguns parâmetros clínicos (distribuição segundo o gênero, idade no início dos sinais e sintomas, na $1^{\text {a }}$ consulta e no diagnóstico da doença, principais sinais e sintomas e estado nutricional) e laboratoriais da $1^{\text {a }}$ consulta, que permitiram o diagnóstico (enema opaco, manometria anorretal e biópsia retal), bem como a evolução dos pacientes após o tratamento cirúrgico. Resultados: 42 crianças $(79,2 \%)$ eram meninos. Os primeiros sinais e sintomas ocorreram no $1^{0}$ mês, em 43 casos $(81,1 \%)$, a idade, na primeira consulta, teve mediana de 7 meses e idade de diagnóstico, mediana de 8,8 meses. As principais manifestações clínicas foram: distensão abdominal em 83,0\%, constipação intestinal em 73,6\%, atraso na eliminação do mecônio em $60,7 \%$ dos casos. Não foi evidenciado importante comprometimento ponderoestatural. O enema opaco mostrou zona de transição em 47,2\% (17/36) e, na manometria anorretal, observou-se ausência de reflexo reto esfincteriano em $73,7 \%$ (14/19). Todas as biópsias anorretais evidenciaram aganglionose. Uma das crianças foi submetida a tratamento clínico e 52 a tratamento cirúrgico. A colostomia foi realizada em 22/52 crianças (42,3\%) e o tratamento cirúrgico definitivo em 49 , sendo, em $77,6 \%$, cirurgia de Duhamel Haddad, em $20,4 \%$, cirurgia de Soave e, em 2,0\%, cirurgia de Lestar Martin. As complicações pré-operatórias mais freqüentes foram obstrução intestinal em $28,3 \%$ e enterocolite em $11,3 \%$ dos casos. As principais complicações pós operatórias da colostomia foram: eventração e/ou prolapso e/ou estenose em $22,7 \%$ dos casos e do tratamento cirúrgico definitivo: escape e/ou incontinência fecal em $28,6 \%$, infecção e/ou deiscência de ferida cirúrgica em $22,4 \%$ e enterocolite e/ou diarréia em $20,4 \%$. Três crianças não foram submetidas a tratamento cirúrgico definitivo. Dez crianças $(18,9 \%)$ obtiveram alta, $14(26,4 \%)$ continuam em seguimento ambulatorial e $27(50,9 \%)$ abandonaram o serviço após a cirurgia definitiva. Houve um óbito devido a enterocolite algum tempo após a cirurgia definitiva e 1 criança continua em seguimento ambulatorial em outro serviço, após a reconstrução do trânsito. Cinco das 14 crianças (35,7\%) que permanecem em seguimento e $8(29,6 \%)$ das 27 crianças que abandonaram o serviço mantinham escape e/ou incontinência fecal na última consulta. Conclusão: houve uma discrepância entre a idade de início dos sintomas e a idade de diagnóstico, evidenciando retardo no encaminhamento dos pacientes para comprovação diagnóstica. O diagnóstico tardio, possivelmente, determinou a alta porcentagem das complicações pré-operatórias da doença, que permanecem com as principais causas de morbimortalidade relativa à doença.

UNITERMOS: Doença de Hirschsprung. Constipação. Motilidade Gastrointestinal. Criança. 


\section{INTRODUÇÃO}

O megacólon congênito, também conhecido como doença de Hirschsprung, em homenagem a Harald Hirschsprung, que primeiramente descreveu tal desordem em recém-nascidos com constipação grave associada à dilatação e hipertrofia do colón ${ }^{(1,2)}$, é caracterizado pela ausência de células ganglionares nos plexos mientéricos do tracto intestinal distal ${ }^{(3,4)}$. A ausência, no reto, dos gânglios nervosos de Auerbach e de Meissner estende-se em sentido proximal (cranial), desde o ânus até distâncias variáveis dos cólons, às vezes podendo atingir o intestino delgado ${ }^{(5)}$. Aproximadamente 80 a $90 \%$ dos casos são classificados como doença de Hirschsprung de segmento curto, afetando a região retosigmóide distalmente, $25 \%$ do grupo são de doença de Hirschsprung de segmento ultra-curto, acometendo a região anorretal distal. O megacólon congênito de segmento longo é raro e pode se estender para o intestino delgado ${ }^{(3,6)}$.

Fisiopatologicamente, o segmento desnervado atua como lesão obstrutiva, pela sua incapacidade de transmitir as ondas peristálticas que vêm da parte superior do intestino, impedindo, assim, a passagem de fezes através do reto ${ }^{(5)}$.

A incidência varia de $1 / 2000$ a $1 / 5000$ nascidos vivos, acometendo o sexo masculino em 70 a $80 \%$ dos $\operatorname{casos}^{(2,6)}$. No recém-nascido, freqüentemente se apresenta como quadro de abdômen agudo obstrutivo ou constipação intestinal acompanhada de distensão abdominal e diarréia paradoxal. Já na criança maior, a forma mais comum de apresentação é a de constipação intestinal crônica ${ }^{(2,5)}$.

O diagnóstico do megacólon congênito deve ser suspeitado em presença de quadro clínico compatível, como: atraso na eliminação de mecônio, constipação intestinal, distensão abdominal, obstrução intestinal, toque retal evidenciando diminuição do diâmetro do reto (espasmo retal), e à retirada do dedo, eliminação explosiva de fezes líqüidas e gazes. Os exames laboratoriais que auxiliam no diagnóstico são: manometria anorretal (com ausência do reflexo retoesfincteriano), enema opaco (com zona de transição) e biópsia retal (com ausência de neurônios nos plexos de Meissner e Auerbach e teste da acetilcolinesterase positivo) ${ }^{(7 / 11)}$.

$\mathrm{O}$ tratamento é sempre cirúrgico, visando à retirada da parte agangliônica e à restauração da continuidade do intestino, sendo as técnicas cirúrgicas mais comumente utilizadas a de Soave e a de Duhamel ${ }^{(3)}$.

Neste estudo, foi realizado o levantamento de crianças com doença de Hirschsprung, analisando-se algumas características clínicas e laboratoriais, visando alertar o pediatra para a necessidade de encaminhar as crianças o mais precocemente, com o intuito de diminuir as complicações advindas da doença não tratada

\section{MÉTODOS}

Foi realizado estudo retrospectivo mediante revisão de prontuários de todas as 53 crianças com doença de Hirschsprung, atendidas nos serviços de Gastroenterologia e Cirurgia Pediátricas e Proctologia do Hospital das Clínicas de Ribeirão Preto da FMRP/ USP, no período de janeiro de 1981 a dezembro de 1999.

Os dados colhidos foram os relacionados a seguir.

a) Dados de anamnese - distribuição das crianças segundo o gênero, idade das crianças no início dos sinais e sintomas, na $1^{\text {a }}$ consulta e no diagnóstico da doença, e principais sinais e sintomas da doença. Na avaliação do estado nutricional, foi utilizado o escore $Z^{(12)}$, tomando-se como referência a curva do NCHS ${ }^{(13)}$.

b) Dados de exames laboratoriais - algumas características evidenciadas no enema opaco, manometria anorretal e biópsia retal. O enema opaco foi realizado pela técnica de Neuhauser ${ }^{(9)}$ sem a realização de preparo intestinal e/ou lavagens intestinais por, pelo menos, $48 \mathrm{~h}$ antes do exame, para facilitar a visualização da existência de zona de transição. Para a manometria anorretal foi utilizada uma sonda de polivinil, tendo três canais com aproximadamente $2,4 \mathrm{~mm}$ de diâmetro externo e $1,0 \mathrm{~mm}$ de diâmetro interno. Os dois orifícios laterais distavam $1,6 \mathrm{~cm}$ entre si e eram perfundidos com água a $0,25 \mathrm{ml} / \mathrm{min}$, por um sistema de perfusão montado no próprio serviço. A distensão retal foi induzida por um balão de látex amarrado na extremidade da sonda, $4,5 \mathrm{~cm}$ acima do primeiro orifício lateral. As pressões era captadas por transdutores situados em cada linha de perfusão e conectados a pré amplificadores do registrador (Poligrafo Physiograph MK-IV, Narco BioSystems- a division of Narco Scientific), por onde era obtido o traçado em papel. As pressões dos esfíncteres anais, interno e externo, foram localizadas através da técnica do pull through, com 
retirada contínua da sonda, estando a criança em decúbito lateral direito ${ }^{(7,8)}$. A biópsia retal foi realizada pela técnica de Swenson ${ }^{(10,11)}$, sendo os cortes obtidos a $2 \mathrm{~cm}$ da linha pectínea. Não foi feita a técnica da acetilcolinesterase em nenhuma delas.

c) Dados de tratamento - tipo de tratamento cirúrgico realizado, bem como a evolução dos pacientes após a correção cirúrgica, destacando as principais complicações apresentadas pelos mesmos.

\section{RESULTADOS}

Das 53 crianças analisadas, $42(79,25 \%)$ eram do sexo masculino e $11(20,75 \%)$, do feminino.

A idade na 1a. consulta variou de um dia a 19 anos (mediana de sete meses).

O quadro clínico se iniciou no primeiro mês de vida em 43 casos $(81,1 \%)$. A mediana da idade das crianças, no diagnóstico, foi de oito meses e 25 dias (Figura 1).
As principais manifestações clínicas foram: distensão abdominal em 83,0\%, constipação intestinal em $73,6 \%$, atraso na eliminação de mecônio (acima de 24h) em 60,7\%, vômitos em 43,4\%, obstrução intestinal em $28,3 \%$, diarréia em $13,2 \%$, enterocolite em $11,3 \%$ e perfuração intestinal em $3,8 \%$.

Foram analisados o peso de 51 crianças e a estatura de 42 crianças, observando-se comprometimento ponderoestatural em aproximadamente $21 \%$ delas (Escore $\mathrm{Z}$ para peso e estatura $\square \square 2$ ).

$\mathrm{Na}$ investigação foram realizados 36 enemas opacos, dos quais 17 (47,2\%) evidenciaram zona de transição e 31 (86,1\%) dilatação e/ou redundância de alças intestinais. Apenas 19 crianças foram submetidas à manometria anorretal, sendo detectada ausência de reflexo retoesfincteriano em 14 (73,7\%). Todos os pacientes foram submetidos à biópsia retal, que mostrou ausência de neurônios nos plexos de Meissner e Auerbach, nos segmentos analisados.

Das 53 crianças, uma foi submetida a tratamento clínico e 52 a tratamento cirúrgico. A colostomia e/ou ileostomia foram realizadas em $22 / 52(42,3 \%)$ e o

\section{Idade de ap ar ecimento dos primeir os sinais/sintomas}

\section{Idade de diagnóstico}

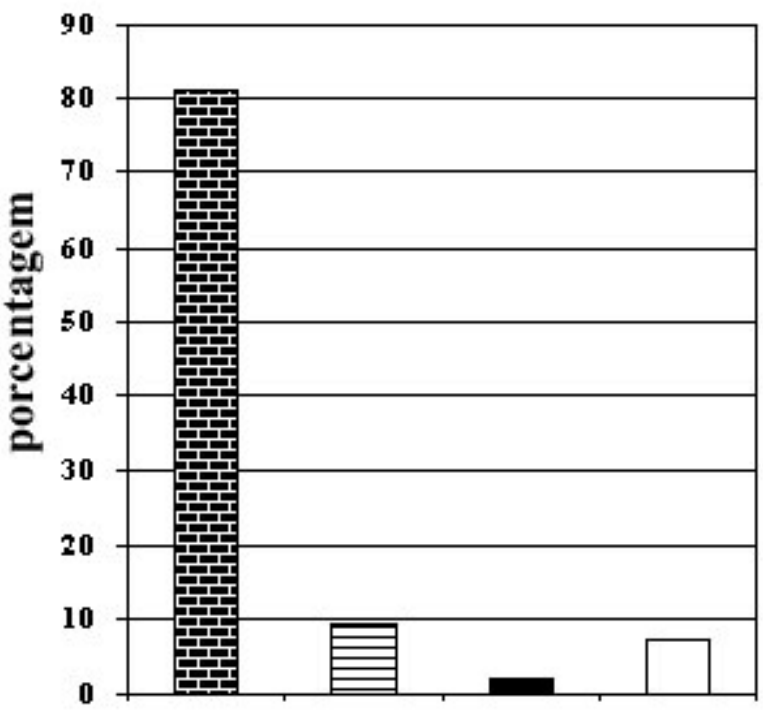

$0-1 m \quad 1 m-6 m \quad 6 m-1 a>1 a$

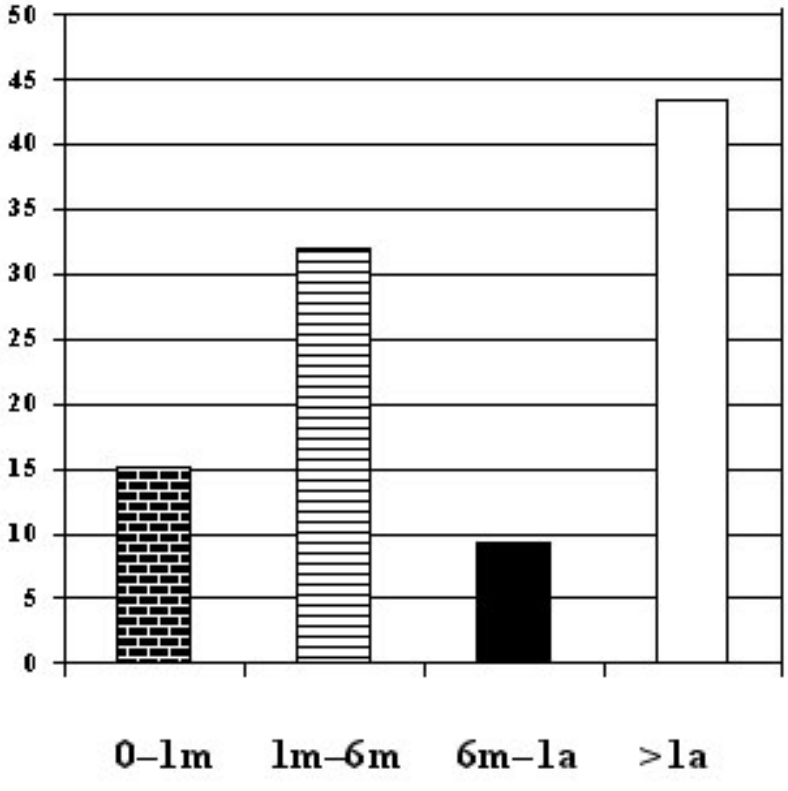

Figura 1 - Idade de aparecimento dos primeiros sinais e sintomas e idade do diagnóstico da doença de Hirschsprung, em 53 crianças. 
tratamento cirúrgico definitivo em 49 casos, sendo utilizada a cirurgia de Duhamel Haddad em 38/49 (77,6\%), a cirurgia de Soave em 10/49 (20,4\%) e, em 1/49 $(2,0 \%)$, a cirurgia de Lestar Martin.

Ocorreram, como principais complicações póscirúrgicas da colostomia, eventração e/ou prolapso e/ ou estenose em $5 / 22(22,7 \%)$ dos casos, e do tratamento cirúrgico definitivo, escape e/ ou incontinência fecal em 14/49 (28,6\%), infecção e/ou deiscência de ferida cirúrgica em 11/49 (22,4\%) e enterocolite e/ou diarréia em 10/49 (20,0\%). Três crianças $(5,7 \%)$ não foram submetidas a tratamento cirúrgico definitivo (uma abandonou o serviço antes de qualquer tratamento cirúrgico, uma após a colostomia e a outra fez colostomia e aguarda tratamento cirúrgico definitivo).

No seguimento das 53 crianças, dez $(18,9 \%)$ obtiveram alta, $14(26,4 \%)$ continuam em seguimento ambulatorial e $27(50,9 \%)$ abandonaram o serviço. Houve um óbito devido a enterocolite, algum tempo após a cirurgia definitiva, e uma criança continuou seguimento ambulatorial em outro serviço após reconstrução do trânsito. Cinco crianças $(35,71 \%)$ que permanecem em seguimento e oito, $(29,6 \%$ das crianças) que abandonaram o serviço mantinham escape fecal/ incontinência fecal na última consulta .

\section{DISCUSSÃO}

Evidenciou- se, neste estudo, predomínio do sexo masculino para a doença de Hirschsprung, na proporção de $3,8: 1$, achado concordante com a literatura que mostra razões meninos/meninas, variando de $2: 1$ a $4: 1$ $(1,3,4,6,14 / 18)$.

Observou- se atraso no encaminhamento e, conseqüentemente, no diagnóstico da doença. Em aproximadamente $80 \%$ dos casos, as manifestações clínicas iniciaram- se no $1^{\text {o. }}$ mês de vida, e a idade, na $1^{\text {a }}$ consulta, teve mediana de sete meses. Aproximadamente, metade das crianças teve o diagnóstico confirmado após os seis meses de idade. Na literatura, a maioria dos autores refere o diagnóstico da doença no $1^{\circ}$. mês de vida em 50 a $60 \%$ dos casos, idade muito mais precoce que na casuística presente (Tabela I). $\mathrm{O}$ retardo entre o início dos sinais e sintomas e a investigação da doença aumenta o risco de complicações, sendo a mais importante a enterocolite e, conseqüentemente, aumentando a morbimortalidade do megacólon congênito ${ }^{(14,19)}$.

As manifestações clínicas mais freqüentes foram a distensão abdominal, a constipação intestinal, os vômitos e o atraso na eliminação do mecônio. Isso também foi verificado por Jung, em $1995^{(15)}$, quando avaliando a apresentação clínica da doença de Hirschsprung em crianças no período neonatal, encontrou distensão abdominal em $90,0 \%$, vômitos em $67,1 \%$, constipação em 23,0\%, e atraso na eliminação do mecônio (após 48h de vida) em 51,0\% dos casos. Já em crianças após o período neonatal, ocorria constipação em $68,7 \%$ dos casos, distensão abdominal em $64,2 \%$, vômitos em $37 \%$ e atraso na eliminação de mecônio em 40,6\%. A maioria dos pesquisadores encontraram, como principais formas de apresentação da doença de Hirschsprung, a obstrução intestinal e a constipação intestinal, sendo que a obstrução é mais comum no período neonatal, ocorrendo em 42 a $81 \%$ dos casos e a constipação intestinal acima de um mês de idade, ocorrendo em 13 a $69 \%$ das crianças ${ }^{(3,15,17,18)}$.

Neste estudo, a constipação intestinal foi mais freqüente que os quadros de obstrução intestinal, o que, provavelmente, seja decorrente do fato de que a maioria das crianças que procuraram o serviço para investigação, apresentavam mais de um mês de vida.

\begin{tabular}{|c|c|c|c|c|}
\hline \multirow[t]{2}{*}{ AUTORES } & \multicolumn{4}{|c|}{ IDADE DO DIAGNÓSTICO } \\
\hline & $=1$ mês & \multicolumn{2}{|c|}{$1 m-1$ ano } & $=1$ ano \\
\hline MARTY et al. (1995) & $64 \%$ & \multicolumn{2}{|c|}{$21 \%$} & - \\
\hline JUNG (1995) & $51 \%$ & \multicolumn{2}{|c|}{$31 \%$} & $18 \%$ \\
\hline \multirow[t]{2}{*}{ FORTUNA et al. (1996) } & $57 \%$ & \multicolumn{2}{|c|}{$27 \%$} & $16 \%$ \\
\hline & $<1$ mês & $1 m-6 m$ & $6 m-1$ ano & $=1$ ano \\
\hline TEITELBAUM et al. (1988) & $64 \%$ & $18 \%$ & $18 \%$ & - \\
\hline Este estudo & $15 \%$ & $32 \%$ & $9 \%$ & $43 \%$ \\
\hline
\end{tabular}


Em 2/3 das crianças para as quais se questionou sobre a primeira evacuação após o nascimento, foi comprovado o atraso na eliminação do mecônio, destacando-se a importância da observação, já no berçário, do hábito intestinal do recém-nascido, o que possibilitou a suspeita e, conseqüentemente, o diagnóstico precoce do megacólon congênito.

Detectou- se déficit ponderal e/ ou estatural em aproximadamente $21 \%$ dos casos, sendo reforçada, mais uma vez, a importância do diagnóstico precoce, a fim de ser evitado o comprometimento do crescimento e desenvolvimento da criança.

Os enemas opacos foram altamente sugestivos de doença de Hirschsprung ou seja, com zona de transição em aproximadamente $50 \%$ dos casos, isso também foi verificado por Junqueira \& Matushita ${ }^{(9)}$. A baixa porcentagem observada, provavelmente, deveuse ao fato de somente ter sido considerada zona de transição aquela em que havia uma área bem definida de estreitamento na imagem radiológica. Sabe-se entretanto, que o encontro de alças colônicas proximais, de maior diâmetro que as distais, já é o suficiente para o diagnóstico de zona de transição. A manometria anorretal evidenciou ausência de reflexos em aproximadamente $73,7 \%$ dos pacientes. Na literatura, há dados de que a sensibilidade do enema opaco esteja em torno de $80 \%$ e a da manometria anorretal, em torno de $79 \%{ }^{(17)}$.

Em 4 manometrias, observou-se o reflexo retoesfincteriano. Como já descrito anteriormente, a presença desse reflexo descarta o diagnóstico de megacólon congênito, tornando desnecessários exames adicionais. Embora confiável, quando bem realizada, a manometria requer equipamento relativamente caro e treinamento especial, para distinguir respostas significativas de $\operatorname{artefatos}^{(2)}$. Possivelmente, as manometrias, em que foram descritas presença de reflexo, foram mal interpretadas, pois na época, o procedimento manométrico estava sendo implantado no serviço e havia dificuldades na técnica e na interpretação desse exame.

A biópsia retal é considerada o exame padrãoouro para o diagnóstico do megacólon congênito. $\mathrm{O}$ enema opaco e a manometria anorretal não são suficientes como método único na avaliação da criança constipada. A manometria anorretal é superior ao enema opaco na avaliação do segmento ultracurto, na aganglionose total e em pacientes com colostomia ${ }^{(9,20,}$ 21). Entretanto, a associação da manometria anorretal com o enema opaco propicia acurácia de $100 \%$ no diagnóstico da doença de Hirschsprung, quando há concordância entre os métodos. Sendo assim, a biópsia poderia ser reservada para os casos em que não houver concordância entre o enema opaco e manometria, ou seja, quando existe dúvida no diagnóstico ${ }^{(9)}$.

No serviço assim como na literatura ${ }^{(3)}$, as técnicas cirúrgicas mais utilizadas para a reconstrução do trânsito intestinal foram as descritas por Duhamel Haddad e Soave.

As complicações pós-cirúrgicas mais freqüentemente encontradas foram a incontinência/ escape fecal, a infecção/ deiscência da ferida cirúrgica e enterocolite/ diarréia. A incontinência fecal é sintoma freqüente em crianças com megacólon congênito após reconstrução cirúrgica. Catto-Smith et al., em 1995 (22), observaram que $80 \%$ das crianças com tratamento cirúrgico da doença de Hirschsprung tinham incontinência, sendo que, em 53\%, a incontinência era bastante significativa, segundo a avaliação dos pais das crianças. A enterocolite é a complicação pós-cirúrgica mais temida. Os fatores de risco do desenvolvimento da enterocolite pós operatória ainda são pouco entendidos. Embora a reconstrução cirúrgica resolva, com sucesso, os sintomas obstrutivos do megacólon congênito, ela não elimina o risco do desenvolvimento da enterocolite, que pode ocorrer em 19,0 a $32,0 \%$ dos $\operatorname{casos}^{(1,3,23)}$.

Após o tratamento cirúrgico, observou- se índice de cura (analisada pelas altas ambulatoriais) bastante baixo, menor que $20 \%$. A maioria das crianças que estão em seguimento ambulatorial têm queixa de escape/incontinência fecal.

Nota-se, portanto, que mesmo retirando o segmento comprometido, não se estabelece a recuperação total dos pacientes, tornando-se necessário entender melhor os mecanismos fisiopatológicos da doença de Hirschsprung para aprimorar as técnicas terapêuticas, a fim de permitir a cura total do paciente, diminuindo o risco de seqüelas.

Pelos presentes dados, enfatiza-se a necessidade de alertar o pediatra geral para o conhecimento do quadro clínico da doença de Hirschsprung bem como de suas complicações e a importância do diagnóstico precoce da doença. 
BIGÉLLI RHM; FERNANDES MIM; GALVÃO LC \& SAWAMURA R. Retrospective study of 53 children with Hirschsprung's disease: clinical and laboratorial findings. Medicina, Ribeirão Preto, 35: 78-84, jan./march 2002.

ABSTRACT: Objective: to analyze some clinical and laboratory characteristics in children with Hirschsprung's disease. Methods: a retrospective study of all 53 children with Hirschsprung's disease attended at the University Hospital, Faculty of Medicine of Ribeirão Preto, USP, from January 1981 to December 1999. Some clinical parameters were investigated at the first visit (patients distribution by gender, age at onset of signs and symptoms, at the first visit and at the time of diagnosis, main signs and symptoms, and nutritional status), as well as laboratory tests that permitted the diagnosis (some characteristics observed by barium enema, anorectal manometry and rectal biopsy) and the evolution of the patients after surgical treatment. Results: 42 children $(79.2 \%)$ were boys. The first signs and symptoms occurred during the first month of life in 43 cases $(81.1 \%)$, the average age at the first visit was 7 months and age at the time of diagnosis was 8.8 months. The main clinical manifestations were: abdominal distension in $83.0 \%$, intestinal constipation in $73.6 \%$, delayed passage of meconium in $60.7 \%$. No important deficit in weight and height was observed. A barium enema showed a transition zone in $47.2 \%(17 / 36)$ and anorectal manometry revealed the absence of the relaxation reflex of the internal anal sphincter in $73.7 \%$ (14/19). All anorectal biopsies demonstrated aganglionosis. One child was submitted to clinical treatment and 52 to surgical treatment. Colostomy was performed in $22 / 52$ children (42.3\%) and definitive surgical treatment in 49 (Duhamel Haddad surgery in $77.6 \%$, Soave surgery in $20.4 \%$ and Lestar Martin surgery in $2.0 \%$ ). The most frequent complications before surgery were intestinal obstruction in $28.3 \%$ and enterocolitis in $11.3 \%$. The major complications after colostomy were eventration and/or prolapse and/or stenosis in $22.7 \%$, and the major complications after definitive surgical treatment were soiling and/ or fecal incontinence in $28.6 \%$, infection and/or dehiscence of the surgical wound in $22.4 \%$, and enterocolitis and/or diarrhea in $20.0 \%$. Three children were not submitted to surgical treatment. Ten (18.9\%) received ambulatory discharge, 14 $(26.0 \%)$ are still being followed up and $27(50.9 \%)$ abandoned the service. There was a death $(1.9 \%)$ by enterocolitis some time after definitive surgical treatment and one child $(1.9 \%)$ is still being followed up in another service, after reconstruction of the transit. Conclusions: there was a discrepancy between the age at onset of symptoms and the age at diagnosis, demonstrating a delay in patient referral for diagnostic confirmation. The late diagnosis probably determined the high percentage of presurgical complications of Hirschsprung's disease, i.e.,: intestinal obstruction $(28.3 \%)$ and enterocolitis $(11.3 \%)$ which are the most frequent and continue to be the major causes of morbidity and mortality in this disease.

UNITERMS: Hirschsprung's Disease. Megacolon. Constipation. Gastrointestinal Motility. Child.

\section{REFERÊNCIAS BIBLIOGRÁFICAS}

1 - MARTY TL; SEO T; MATLAK ME; SULLIVAN JJ; BLACK RE JOHNSON DG. Gastrointestinal function after surgical correction of Hirschsprung's disease: long term follow up in 135 patients. J Pediatr Surg 30: 655-658, 1995.

2 - RUDOLPH C \& BENAROCH L. Hirschsprung disease. Pediatr Rev 16: 5-11, 1995.

3 - FORTUNA RS; WEBER TR; TRACY JR TF; SILEN ML \& CRADOCK TV. Critical analysis of the operative treatment of Hirschsprung's disease. Arch Surg 131: 520-525, 1996.

4 - RAJAB A; FREEMAN NV \& PATTON MA. Hirschsprung's disease in Oman. J Pediatr Surg 32: 724-727, 1997.

5 - BRAZ A. Megacólon. Considerações fisiopatológicas, diagnósticas e terapêuticas acerca da moléstia de
Hirschsprung e da obstrução intestinal crônica. Pediatr Mod 20: 277-304, 1985.

6 - QUALMAN SJ \& MURRAY R. Aganglionosis and related disorders. Hum Patol 25: 1141-1149, 1994.

7 - MEUNIER PD. Anorectal manometry. A collective international experience. Gastroenterol Clin Biol 15: 697-702, 1991.

8 - MEUNIE PD \& GALLAVARDIN D. Anorectal manometry: the state of the art. Dig Dis 11: 252-264, 1993.

9 - JUNQUEIRA MA \& MATUSHITA JPK. Acuidade do enema opaco pela técnica de Neuhauser no diagnóstico da doença de Hirschsprung. Radiol Bras 24: 113-116, 1991.

10 - SWENSON O; FISHER JH \& MACMAHON E. Rectal biopsy as an aid in the diagnosis of Hirschsprung's disease. $\mathbf{N}$ Engl $\mathbf{J}$ Med 253: 632-635, 1955 
11 - GUGELMIN ES; TORRES LFB \& SABBAGA CC. Aplicação da biópsia de reto por sucção no diagnóstico da doença de Hirschsprung. J Pediatr 66: 171-174, 1990.

12 - BARROS FC; VICTORA CG \& SHRIMPTON R (1991). Indicadores de saúde. In: BARROS FC \& VICTORA CG, eds. Epidemiologia da saúde infantil. Um manual para diagnósticos comunitários. Editora Hucitec, Unicef, São Paulo.

13 - KUCZMARSKI RJ; OGDEN CL; GRUMMER-STRAWN LM; FLEGAL MS; GUO SS; WEI R; MEI Z; CURTIN LR; ROCHE AF \& JOHNSON CL. CDC growth charts: United States. Adv Data 314: 1-27, 2000.

14 - TEITELBAUM DH; QUALMAN S \& CANIANO DA. Hirschsprung's disease. Identification of the risk factors for enterocolitis. Ann Surg 207: 240-244, 1988.

15 - JUNG PM. Hirschsprung's disease: one surgeon's experience in one institution. J Pediatr Surg 30: 646-651, 1995.

16 - ELHALABY EA; TEITELBAUM DH; CORAN AG \& HEILDELBERGER KP. Enterocolitis associated with Hirschsprung's disease: a clinical histopathological correlative study. J Pediatr Surg 30: 1023-1027, 1995.

17 - REDING R; DEGOYET SDV; GOSSEYE S; CLAPUYT P; SOKAL E; BUTS JP; GIBBS P \& OTTE JB. Hirschsprung's disease: a 20 years experience. J Pediatr Surg 32: 12211225, 1997.
18 - YANCHAR NL \& SOUCY P. Long- term outcome after Hirschsprung's disease: patients' perspectives. J Pediatr Surg 34: 1152-1160, 1999.

19 - ELHALABY EA; CORAN AG; BLANE CE; HIRSCHI RB; TEITELBAUM DH \& ARBOR A. Enterocolitis associated with Hirschsprung's disease: a clinical radiological characterization based on 168 patients. J Pediatr Surg 30: 76-83, 1995.

20 - BAKER SS; LIPTAK GS; COLLETTI RB; CROFFIE JM; DILORENZO C; ECTOR W \& NURKO S. Constipation in infants and children: evaluation and treatment. J Pediatr Gastroenterol Nutr 29: 612-626, 1999.

21 - DA-COSTA-PINTO EAL; BUSTORFF- SILVA JM \& FUKUSHIMA, E. Manometria anorretal e diagnóstico diferencial da constipação em crianças. J Pediatr 76: 227-232, 2000.

22 - CATTO-SMITH AG; COFFEY CMM; NOLAN TM \& HUTSON JM. Fecal incontinence after the surgical treatment of Hirschsprung disease. J Pediatr 127: 954-957, 1995.

23 - HACKAM DJ; FILLER RM \& PEARL RH. Enterocolitis after the surgical treatment of Hirschsprung's disease: risk factors and financial impact. J Pediatr Surg 33: 830-833, 1998.

Recebido para publicação em 26/06/2001

Aprovado para publicação em 27/03/2002 